\title{
Editorial
}

\section{Depressive Symptoms in Persons with Epilepsy: Methodological Issues}

Epilepsy is considered to be the most common serious neurological ailment, which is associated with several adverse emotional, behavioral, social, and cognitive consequences and overall poor quality of life. ${ }^{[1,2]}$ Psychiatric comorbidity is reported to be quite common among patients with epilepsy, and is known to have bidirectional relationship with epilepsy it can precede the onset, accompany or follow the diagnosis of epilepsy, and contribute to overall poor quality of life of the patient. ${ }^{[3]}$ Hence, it is very important to recognize and treat the psychiatric disorders in patients with epilepsy.

Although almost all psychiatric disorders have been documented in patients with epilepsy, depression has been shown to be the most common psychiatric disorders among patients with epilepsy. The prevalence of depression in patients with epilepsy has been estimated by different studies across the world, and there is a wide variation in the estimate. The wide variation is attributed to differences in the study designs, demographic variable, and most importantly the method used to diagnose depression or epilepsy. ${ }^{[4]}$ A meta-analysis of available studies reported the prevalence of current depression or depression in the past 1 year to be $23.1 \%$ with an odds ratio of 2.77 for active depression in persons with epilepsy. ${ }^{[4]}$

One of the important variables, which influences the wide variation in the prevalence of depression is the assessment method. As it is not possible for every patient to be evaluated by a mental health professional to estimate the prevalence of mental disorders, researchers have relied upon various screening instruments to assess depression. A recent systematic review evaluated all the available studies and reported that 16 screening instruments have been validated to assess depression among patients with epilepsy. These tools have been validated in 13 languages. Among the various tools, the screening instrument, which has been most commonly used, is the Neurological Disorders Depression Inventory for Epilepsy (NDDI-E). Unfortunately, NDDI-E is not validated in any Indian language..$^{[5]}$ Other commonly used rating scales include Beck Depression Inventory, Hospital Anxiety and Depression Scale, Emotional Thermometers, Patient Health Questionnaire-9 (PHQ-9), PHQ-2, and the Hamilton Rating Scale for Depression. ${ }^{[5]}$ In this systematic review, authors pointed out that diagnostic accuracy of various screening instruments has been reported inconsistently and often the cut points used to diagnose depression have been selected post hoc. ${ }^{[5]}$ The recommended cutoff for PHQ-9 is 10 , with a sensitivity of $92 \%$ and specificity of $74 \%$. ${ }^{[5]}$
The gold standard for estimation of depression or any psychiatric disorder is an evaluation of a person by a trained/qualified psychiatrist using a structured validated instrument. Many diagnostic instruments have been used to evaluate the prevalence of depression among persons with epilepsy, with Mini International Neuropsychiatric Interview (MINI) being the most preferred instrument. ${ }^{[5]}$

Occasional clinic-based studies from India in the past have evaluated the prevalence of depression in persons with epilepsy and estimated it to be $5 \%{ }^{[6]}$ Other clinic-based studies, although evaluated depression did not report the prevalence rate. ${ }^{[7,8]}$ However, on the basis of correlation analysis concluded that quality of life of patients with epilepsy is strongly influenced by depression and seizure frequency. ${ }^{[7,8]}$ Accordingly, there is a need to study this area further in the Indian context.

Accordingly, the study on depressive symptoms in persons with epilepsy published in this issue of the journal is a welcome step. ${ }^{[9]}$ In this study, the authors evaluated 150 adult patients with epilepsy using Tamil version of PHQ-12. Authors reported the prevalence of depressive symptoms in about two-third (63.3\%) of the patients, and in the multivariate regression, analysis the significant predictors of depressive symptoms were secondary generalized seizures (odds ratio 5.48) and seizure frequency $>1$ /month (odds ratio 2.53). ${ }^{[9]}$ Although this study adds to the limited literature from India on depression in persons with epilepsy, there are certain methodological issues, which need to be considered in the interpretation of this study.

First, the authors calculated the sample size of 148 on the basis of $30 \%$ prevalence of depression. On the face value, this may appear to be methodologically sound, but, what is important to understand is that the prevalence figure pertains to depression and not depressive symptoms. Because it is well known that many patients with various physical disorders can have subsyndromal depressive symptoms and overall prevalence of symptoms would be always more than that of syndromal depression, which is always considered to have a threshold level, above which a disorder is considered to be present or absent. Accordingly, the authors committed error in calculating their sample size, which could be much larger than what was estimated by the author.

Second, authors used Tamil version of PHQ-12 to estimate depressive symptoms. The PHQ-12 as reported by the authors was a modification of PHQ-9 questionnaire and this involved splitting of three items of PHQ-9 
into 2, which resulted in PHQ-12. Further, in the index study, the authors changed the response categories to dichotomous responses (yes and no) and used a cutoff of $>4$ to define depression. Although the authors claim that this was done to make the instrument culturally compatible for the Indian setting, they did not provide enough justification for the same. In fact, the original study, which published the modified PHQ, reported that this was done to ease the use of the scale to screen depression in large epidemiological studies. ${ }^{[10]}$ However, such was not true for this study. It is important to note that as per the original PHQ-9 scale, all the items are rated on a four-point scale (0-3) depending on the number of days the symptoms are present. A PHQ-9 score of $\geq 10$ was found have high sensitivity and specificity for major depression. Further, the PHQ-9 scores of 5, 10, 15 , and 20 are used to define mild, moderate, moderately severe, and severe depression, respectively. ${ }^{[11]}$ However, it is important to note that if the aim was to estimate the prevalence of depressive symptoms and not depression, then what was the need to define a cutoff of 4 , because any value above zero, will be suggestive of depressive symptoms. Hence, the prevalence of depressive symptoms reported by the authors is not an accurate estimate of depressive symptoms.

Third, when the authors come to their discussion, again they try to compare the prevalence of depressive symptoms obtained in their study with depression as reported on various scales using various cutoff levels.

It is important to understand that screening instruments are usually used when a large community-based population is to be evaluated, and for clinic-based studies, estimation of psychiatric disorders by mental health professions using standard diagnostic instruments such as MINI should be considered. Future studies must take these methodological issues into consideration while planning research.

Sandeep Grover

Department of Psychiatry, Postgraduate Institute of Medical Education and Research, Chandigarh, India

Address for correspondence: Dr.Sandeep Grover, Department of Psychiatry, Postgraduate Institute of Medical Education and Research, Chandigarh - 160 012, India. E-mail: drsandeepg2002@yahoo.com

\section{REFERENCES}

1. Theodore WH, Spencer SS, Wiebe S, Langfitt JT, Ali A, Shafer PO, et al. Epilepsy in North America: A report prepared under the auspices of the global campaign against epilepsy, the International Bureau for Epilepsy, the International League Against Epilepsy, and the World Health Organization. Epilepsia 2006; 47:1700-22.

2. National Institute for Health and Clinical Excellence. The Epilepsies: The Diagnosis and Management of the Epilepsies in Adults and Children in Primary and Secondary Care: Pharmacological Update of Clinical Guideline 20. London: National Institute for Health and Clinical Excellence: Guidance; 2012.

3. Cianchetti C, Messina P, Pupillo E, Crichiutti G, Baglietto MG, Veggiotti $\mathrm{P}$, et al. The perceived burden of epilepsy: Impact on the quality of life of children and adolescents and their families. Seizure 2015;24:93-101.

4. Fiest KM, Dykeman J, Patten SB, Wiebe S, Kaplan GG, Maxwell CJ, et al. Depression in epilepsy: A systematic review and meta-analysis. Neurology 2013;80:590-9.

5. Gill SJ, Lukmanji S, Fiest KM, Patten SB, Wiebe S, Jetté N. Depression screening tools in persons with epilepsy: A systematic review of validated tools. Epilepsia 2017;58:695-705.

6. Babu CS, Satishchandra P, Sinha S, Subbakrishna DK. Co-morbidities in people living with epilepsy: Hospital based case-control study from a resource-poor setting. Epilepsy Res 2009;86:146-52.

7. Mehta S, Tyagi A, Tripathi R, Kumar M. Study of inter-relationship of depression, seizure frequency and quality of life of people with epilepsy in India. Ment Illn 2014;6:5169.

8. Sirari S, Dhar D, Vishwakarma R, Tripathi S. Association between quality of life, depression and caregiver burden in epileptic patients in Haldwani, Uttarakhand, India. Int J Curr Sci 2014;10:E54-60.

9. Chandrasekharan SC, Menon V, Wadwekar V, Nair PP. High frequency of depressive symptoms among adults with epilepsy: Results from a Hospital-based Study. J Neurosci Rural Pract 2017:13-19.

10. Poongothai S, Pradeepa R, Ganesan A, Mohan V. Reliability and validity of a modified PHQ-9 item inventory (PHQ-12) as a screening instrument for assessing depression in Asian Indians (CURES-65). J Assoc Physicians India 2009;57:147-52.

11. Kroenke K, Spitzer RL, Williams JB. The PHQ-9: Validity of a brief depression severity measure. J Gen Intern Med 2001;16:606-13.

This is an open access article distributed under the terms of the Creative Commons Attribution-NonCommercial-ShareAlike 3.0 License, which allows others to remix, tweak, and build upon the work non-commercially, as long as the author is credited and the new creations are licensed under the identical terms.

\begin{tabular}{|l|l|}
\hline \multicolumn{2}{|c|}{ Access this article online } \\
\hline Quick Response Code: & Website: \\
& \\
\cline { 2 - 2 } & \\
\hline
\end{tabular}

How to cite this article: Grover S. Depressive symptoms in persons with epilepsy: Methodological issues. J Neurosci Rural Pract 2017;8:S3-4. 